Manuscript ID: es-2020-048394

Resubmission to Environmental Science \& Technology 2020-09-19

A new conceptual toxicokinetic model to assess synergistic mixture effects between the aromatic hydrocarbon $\beta$-naphthoflavone and the azole nocodazole on the CYP1A biomarker in a fish cell-line

Shirin Fallahi, Marie Mlnaříková, Charlotte Alvord, Guttorm Alendal, Håvard G. Frøysa, Torbjörn Lundh and Malin C. Celander*

* Corresponding Author, phone: +46 317863693; E-mail: malin.celander@gu.se

\title{
ASSOCIATED CONTENTS
}

\section{Supporting Information}

Number of pages: 15

Number of Figures: 2

Number of Tables: 3

Number of References: 2

\section{TABLES}

Table S1. Raw data for the ethoxyresorufin- $O$-deethylase (EROD) activity in seven different experiments pages S3-7

Table S2. Raw data from a dose response experiment with $\beta$-naphthoflavone (BNF) alone on ethoxyresorufin- $O$-deethylase (EROD) activities at 24 hours in vehicle-control (DMSO) treated cells and in cells treated with $0.1,0.3,0.9,1,3,8$ or $25 \mu \mathrm{M}$ BNF alone pages S8-9

Table S3. Raw data from dose response experiment with nocodazole (NOC) alone on ethoxyresorufin- $O$-deethylase (EROD) activities at 6, 12, 24, 48 and 72h in vehicle-control (DMSO) treated cells and in cells treated with 1,10 or $25 \mu \mathrm{M}$ NOC alone pages $\mathrm{S} 10-12$

\section{FIGURES}

Figure S1. The sensitivity values of the ten parameters at five time points in six different experiments page S13 
Figure S2. Collinearity index for all parameter combinations

page $\mathrm{S} 14$

REFERENCES

page S15 


\section{TABLES:}

Table S1. Raw data for the ethoxyresorufin- $O$-deethylase (EROD) activity shown in Figures 2A, 2B and 4A. All times are given in hours. Treatments are denoted by 1-7. The same annotation is used in the model code. Data are originally published in figures as mean values \pm standard deviation in Gräns et al. 2015 (reference 1) and Michelová M. Master-thesis 2012 (reference 2).

\begin{tabular}{|c|c|c|c|}
\hline $\begin{array}{l}\text { Time } \\
\text { (h) }\end{array}$ & $\begin{array}{l}\text { EROD activity } \\
\left(\mathrm{pmol} \cdot(\mathrm{min} \cdot \mathrm{mg} \text { protein })^{-1}\right)\end{array}$ & Treatment & $\begin{array}{c}\text { Treatment } \\
\text { number }\end{array}$ \\
\hline 6 & 2.92190258 & BNF $0.1 \mu \mathrm{M}$ & 1 \\
\hline 6 & 3.69454764 & BNF $0.1 \mu \mathrm{M}$ & 1 \\
\hline 6 & 1.68262381 & BNF $0.1 \mu \mathrm{M}$ & 1 \\
\hline 6 & 3.3423465 & BNF $0.1 \mu \mathrm{M}$ & 1 \\
\hline 12 & 1.42052688 & BNF $0.1 \mu \mathrm{M}$ & 1 \\
\hline 12 & 2.31785379 & BNF $0.1 \mu \mathrm{M}$ & 1 \\
\hline 12 & 1.18018557 & BNF $0.1 \mu \mathrm{M}$ & 1 \\
\hline 12 & 3.23571203 & BNF $0.1 \mu \mathrm{M}$ & 1 \\
\hline 24 & 0.65168451 & BNF $0.1 \mu \mathrm{M}$ & 1 \\
\hline 24 & 0.5054846 & BNF $0.1 \mu \mathrm{M}$ & 1 \\
\hline 24 & 0.41898238 & BNF $0.1 \mu \mathrm{M}$ & 1 \\
\hline 24 & 0.61937856 & BNF $0.1 \mu \mathrm{M}$ & 1 \\
\hline 48 & 0.05222293 & BNF $0.1 \mu \mathrm{M}$ & 1 \\
\hline 48 & 0.08032718 & BNF $0.1 \mu \mathrm{M}$ & 1 \\
\hline 48 & 0.10036158 & BNF $0.1 \mu \mathrm{M}$ & 1 \\
\hline 48 & 0.03061984 & BNF $0.1 \mu \mathrm{M}$ & 1 \\
\hline 72 & -0.0089312 & BNF $0.1 \mu \mathrm{M}$ & 1 \\
\hline 72 & -0.0431119 & BNF $0.1 \mu \mathrm{M}$ & 1 \\
\hline 72 & 0.03056186 & BNF $0.1 \mu \mathrm{M}$ & 1 \\
\hline 72 & 0.00783302 & BNF $0.1 \mu \mathrm{M}$ & 1 \\
\hline 6 & 2.27896044 & BNF $0.1 \mu \mathrm{M}$ and NOC $1 \mu \mathrm{M}$ & 2 \\
\hline 6 & 2.67142836 & BNF $0.1 \mu \mathrm{M}$ and NOC $1 \mu \mathrm{M}$ & 2 \\
\hline 6 & 2.55450885 & BNF $0.1 \mu \mathrm{M}$ and NOC $1 \mu \mathrm{M}$ & 2 \\
\hline 6 & 4.18042635 & BNF $0.1 \mu \mathrm{M}$ and NOC $1 \mu \mathrm{M}$ & 2 \\
\hline
\end{tabular}


BNF $0.1 \mu \mathrm{M}$ and NOC $1 \mu \mathrm{M}$

BNF $0.1 \mu \mathrm{M}$ and NOC $1 \mu \mathrm{M}$

BNF $0.1 \mu \mathrm{M}$ and NOC $1 \mu \mathrm{M}$

BNF $0.1 \mu \mathrm{M}$ and NOC $1 \mu \mathrm{M}$

BNF $0.1 \mu \mathrm{M}$ and NOC $1 \mu \mathrm{M}$

BNF $0.1 \mu \mathrm{M}$ and NOC $1 \mu \mathrm{M}$

BNF $0.1 \mu \mathrm{M}$ and NOC $1 \mu \mathrm{M}$

BNF $0.1 \mu \mathrm{M}$ and NOC $1 \mu \mathrm{M}$

BNF $0.1 \mu \mathrm{M}$ and NOC $1 \mu \mathrm{M}$

BNF $0.1 \mu \mathrm{M}$ and NOC $1 \mu \mathrm{M}$

BNF $0.1 \mu \mathrm{M}$ and NOC $1 \mu \mathrm{M}$

BNF $0.1 \mu \mathrm{M}$ and NOC $1 \mu \mathrm{M}$

BNF $0.1 \mu \mathrm{M}$ and NOC $1 \mu \mathrm{M}$

BNF $0.1 \mu \mathrm{M}$ and NOC $1 \mu \mathrm{M}$

BNF $0.1 \mu \mathrm{M}$ and NOC $1 \mu \mathrm{M}$

BNF $0.1 \mu \mathrm{M}$ and NOC $1 \mu \mathrm{M}$

BNF $0.1 \mu \mathrm{M}$ and NOC $10 \mu \mathrm{M}$

BNF $0.1 \mu \mathrm{M}$ and NOC $10 \mu \mathrm{M}$

BNF $0.1 \mu \mathrm{M}$ and NOC $10 \mu \mathrm{M}$

BNF $0.1 \mu \mathrm{M}$ and NOC $10 \mu \mathrm{M}$

BNF $0.1 \mu \mathrm{M}$ and NOC $10 \mu \mathrm{M}$

BNF $0.1 \mu \mathrm{M}$ and NOC $10 \mu \mathrm{M}$

BNF $0.1 \mu \mathrm{M}$ and NOC $10 \mu \mathrm{M}$

BNF $0.1 \mu \mathrm{M}$ and NOC $10 \mu \mathrm{M}$

BNF $0.1 \mu \mathrm{M}$ and NOC $10 \mu \mathrm{M}$

BNF $0.1 \mu \mathrm{M}$ and NOC $10 \mu \mathrm{M}$

BNF $0.1 \mu \mathrm{M}$ and NOC $10 \mu \mathrm{M}$

BNF $0.1 \mu \mathrm{M}$ and NOC $10 \mu \mathrm{M}$

BNF $0.1 \mu \mathrm{M}$ and NOC $10 \mu \mathrm{M}$

BNF $0.1 \mu \mathrm{M}$ and NOC $10 \mu \mathrm{M}$

BNF $0.1 \mu \mathrm{M}$ and NOC $10 \mu \mathrm{M}$

BNF $0.1 \mu \mathrm{M}$ and NOC $10 \mu \mathrm{M}$

BNF $0.1 \mu \mathrm{M}$ and NOC $10 \mu \mathrm{M}$ 


\begin{tabular}{|c|c|c|}
\hline 72 & 0.42778956 & BNF $0.1 \mu \mathrm{M}$ and NOC $10 \mu \mathrm{M}$ \\
\hline 72 & 1.18436321 & BNF $0.1 \mu \mathrm{M}$ and NOC $10 \mu \mathrm{M}$ \\
\hline 72 & 1.02342749 & BNF $0.1 \mu \mathrm{M}$ and NOC $10 \mu \mathrm{M}$ \\
\hline 6 & 7.71808341 & BNF $0.1 \mu \mathrm{M}$ and NOC $25 \mu \mathrm{M}$ \\
\hline 6 & 8.32716239 & BNF $0.1 \mu \mathrm{M}$ and NOC $25 \mu \mathrm{M}$ \\
\hline 6 & 7.69936717 & BNF $0.1 \mu \mathrm{M}$ and NOC $25 \mu \mathrm{M}$ \\
\hline 6 & 13.2227151 & BNF $0.1 \mu \mathrm{M}$ and NOC $25 \mu \mathrm{M}$ \\
\hline 12 & 11.8627936 & BNF $0.1 \mu \mathrm{M}$ and NOC $25 \mu \mathrm{M}$ \\
\hline 12 & 12.5949582 & BNF $0.1 \mu \mathrm{M}$ and NOC $25 \mu \mathrm{M}$ \\
\hline 12 & 9.66216727 & BNF $0.1 \mu \mathrm{M}$ and NOC $25 \mu \mathrm{M}$ \\
\hline 12 & 13.5778835 & BNF $0.1 \mu \mathrm{M}$ and NOC $25 \mu \mathrm{M}$ \\
\hline 24 & 10.1189154 & BNF $0.1 \mu \mathrm{M}$ and NOC $25 \mu \mathrm{M}$ \\
\hline 24 & 11.2275265 & BNF $0.1 \mu \mathrm{M}$ and NOC $25 \mu \mathrm{M}$ \\
\hline 24 & 14.5414457 & BNF $0.1 \mu \mathrm{M}$ and NOC $25 \mu \mathrm{M}$ \\
\hline 24 & 17.0127185 & BNF $0.1 \mu \mathrm{M}$ and NOC $25 \mu \mathrm{M}$ \\
\hline 48 & 14.2378479 & BNF $0.1 \mu \mathrm{M}$ and NOC $25 \mu \mathrm{M}$ \\
\hline 48 & 9.99436876 & BNF $0.1 \mu \mathrm{M}$ and NOC $25 \mu \mathrm{M}$ \\
\hline 48 & 10.7615408 & BNF $0.1 \mu \mathrm{M}$ and NOC $25 \mu \mathrm{M}$ \\
\hline 48 & 8.9564097 & BNF $0.1 \mu \mathrm{M}$ and NOC $25 \mu \mathrm{M}$ \\
\hline 72 & 2.44214987 & BNF $0.1 \mu \mathrm{M}$ and NOC $25 \mu \mathrm{M}$ \\
\hline 72 & 2.12808275 & BNF $0.1 \mu \mathrm{M}$ and NOC $25 \mu \mathrm{M}$ \\
\hline 72 & 2.72777848 & BNF $0.1 \mu \mathrm{M}$ and NOC $25 \mu \mathrm{M}$ \\
\hline 72 & 2.03687811 & BNF $0.1 \mu \mathrm{M}$ and NOC $25 \mu \mathrm{M}$ \\
\hline 6 & 4.25915447 & BNF $1 \mu \mathrm{M}$ and NOC $1 \mu \mathrm{M}$ \\
\hline 6 & 4.15426073 & BNF $1 \mu \mathrm{M}$ and NOC $1 \mu \mathrm{M}$ \\
\hline 6 & 4.02362663 & BNF $1 \mu \mathrm{M}$ and NOC $1 \mu \mathrm{M}$ \\
\hline 6 & 6.83484415 & BNF $1 \mu \mathrm{M}$ and NOC $1 \mu \mathrm{M}$ \\
\hline 12 & 6.33964085 & $\mathrm{BNF} 1 \mu \mathrm{M}$ and NOC $1 \mu \mathrm{M}$ \\
\hline 12 & 8.34863858 & BNF $1 \mu \mathrm{M}$ and NOC $1 \mu \mathrm{M}$ \\
\hline 12 & 4.17620219 & BNF $1 \mu \mathrm{M}$ and NOC $1 \mu \mathrm{M}$ \\
\hline 12 & 6.38054672 & $\mathrm{BNF} 1 \mu \mathrm{M}$ and NOC $1 \mu \mathrm{M}$ \\
\hline 24 & 10.405456 & BNF $1 \mu \mathrm{M}$ and NOC $1 \mu \mathrm{M}$ \\
\hline
\end{tabular}




\begin{tabular}{|c|c|}
\hline 24 & 10.771562 \\
\hline 24 & 7.59540869 \\
\hline 24 & 8.8010075 \\
\hline 48 & 2.70204088 \\
\hline 48 & 3.27295458 \\
\hline 48 & 2.8059326 \\
\hline 48 & 1.8645738 \\
\hline 72 & 0.12598001 \\
\hline 72 & 0.19551305 \\
\hline 72 & 0.2272445 \\
\hline 72 & 0.14294592 \\
\hline 6 & 4.05958302 \\
\hline 6 & 4.73479989 \\
\hline 6 & 3.9063292 \\
\hline 6 & 8.25327557 \\
\hline 12 & 9.21418146 \\
\hline 12 & 11.803604 \\
\hline 12 & 7.6698235 \\
\hline 12 & 14.0849049 \\
\hline 24 & 12.7508499 \\
\hline 24 & 13.483253 \\
\hline 24 & 15.9410841 \\
\hline 24 & 19.4159461 \\
\hline 48 & 15.5009285 \\
\hline 48 & 11.9997667 \\
\hline 48 & 11.5037771 \\
\hline 48 & 11.6652826 \\
\hline 72 & 6.55693615 \\
\hline 72 & 5.61974464 \\
\hline 72 & 4.64567565 \\
\hline 72 & 7.22737517 \\
\hline 6 & $4.54633972^{a}$ \\
\hline
\end{tabular}

BNF $1 \mu \mathrm{M}$ and NOC $1 \mu \mathrm{M}$

BNF $1 \mu \mathrm{M}$ and NOC $1 \mu \mathrm{M}$

BNF $1 \mu \mathrm{M}$ and NOC $1 \mu \mathrm{M}$

BNF $1 \mu \mathrm{M}$ and NOC $1 \mu \mathrm{M}$

BNF $1 \mu \mathrm{M}$ and NOC $1 \mu \mathrm{M}$

BNF $1 \mu \mathrm{M}$ and NOC $1 \mu \mathrm{M}$

BNF $1 \mu \mathrm{M}$ and NOC $1 \mu \mathrm{M}$

BNF $1 \mu \mathrm{M}$ and NOC $1 \mu \mathrm{M}$

BNF $1 \mu \mathrm{M}$ and NOC $1 \mu \mathrm{M}$

BNF $1 \mu \mathrm{M}$ and NOC $1 \mu \mathrm{M}$

BNF $1 \mu \mathrm{M}$ and NOC $1 \mu \mathrm{M}$

BNF $1 \mu \mathrm{M}$ and NOC $25 \mu \mathrm{M}$

BNF $1 \mu \mathrm{M}$ and NOC $25 \mu \mathrm{M}$

BNF $1 \mu \mathrm{M}$ and NOC $25 \mu \mathrm{M} \quad 6$

BNF $1 \mu \mathrm{M}$ and NOC $25 \mu \mathrm{M} \quad 6$

BNF $1 \mu \mathrm{M}$ and NOC $25 \mu \mathrm{M} \quad 6$

BNF $1 \mu \mathrm{M}$ and NOC $25 \mu \mathrm{M} \quad 6$

BNF $1 \mu \mathrm{M}$ and NOC $25 \mu \mathrm{M} \quad 6$

BNF $1 \mu \mathrm{M}$ and NOC $25 \mu \mathrm{M} \quad 6$

BNF $1 \mu \mathrm{M}$ and NOC $25 \mu \mathrm{M} \quad 6$

BNF $1 \mu \mathrm{M}$ and NOC $25 \mu \mathrm{M} \quad 6$

BNF $1 \mu \mathrm{M}$ and NOC $25 \mu \mathrm{M} \quad 6$

BNF $1 \mu \mathrm{M}$ and NOC $25 \mu \mathrm{M} \quad 6$

BNF $1 \mu \mathrm{M}$ and NOC $25 \mu \mathrm{M} \quad 6$

BNF $1 \mu \mathrm{M}$ and NOC $25 \mu \mathrm{M} \quad 6$

BNF $1 \mu \mathrm{M}$ and NOC $25 \mu \mathrm{M} \quad 6$

BNF $1 \mu \mathrm{M}$ and NOC $25 \mu \mathrm{M} \quad 6$

BNF $1 \mu \mathrm{M}$ and NOC $25 \mu \mathrm{M} \quad 6$

BNF $1 \mu \mathrm{M}$ and NOC $25 \mu \mathrm{M} \quad 6$

BNF $1 \mu \mathrm{M}$ and NOC $25 \mu \mathrm{M} \quad 6$

BNF $1 \mu \mathrm{M}$ and NOC $25 \mu \mathrm{M} \quad 6$

BNF $1 \mu \mathrm{M}$ and NOC $10 \mu \mathrm{M}$ 


\begin{tabular}{llll}
6 & $5.59647396^{\mathrm{a}}$ & BNF $1 \mu \mathrm{M}$ and NOC $10 \mu \mathrm{M}$ & 7 \\
6 & $5.1440204^{\mathrm{a}}$ & BNF $1 \mu \mathrm{M}$ and NOC $10 \mu \mathrm{M}$ & 7 \\
6 & $9.61475399^{\mathrm{a}}$ & BNF $1 \mu \mathrm{M}$ and NOC $10 \mu \mathrm{M}$ & 7 \\
12 & $6.26397851^{\mathrm{a}}$ & BNF $1 \mu \mathrm{M}$ and NOC $10 \mu \mathrm{M}$ & 7 \\
12 & $8.31189843^{\mathrm{a}}$ & BNF $1 \mu \mathrm{M}$ and NOC $10 \mu \mathrm{M}$ & 7 \\
12 & $6.94923936^{\mathrm{a}}$ & BNF $1 \mu \mathrm{M}$ and NOC $10 \mu \mathrm{M}$ & 7 \\
12 & $12.9031626^{\mathrm{a}}$ & BNF $1 \mu \mathrm{M}$ and NOC $10 \mu \mathrm{M}$ & 7 \\
24 & $6.41179367^{\mathrm{a}}$ & BNF $1 \mu \mathrm{M}$ and NOC $10 \mu \mathrm{M}$ & 7 \\
24 & $7.78904675^{\mathrm{a}}$ & BNF $1 \mu \mathrm{M}$ and NOC $10 \mu \mathrm{M}$ & 7 \\
24 & $13.1874913^{\mathrm{a}}$ & BNF $1 \mu \mathrm{M}$ and NOC $10 \mu \mathrm{M}$ & 7 \\
24 & $15.1788122^{\mathrm{a}}$ & BNF $1 \mu \mathrm{M}$ and NOC $10 \mu \mathrm{M}$ & 7 \\
48 & $4.5062918^{\mathrm{a}}$ & BNF $1 \mu \mathrm{M}$ and NOC $10 \mu \mathrm{M}$ & 7 \\
48 & $2.3500834^{\mathrm{a}}$ & BNF $1 \mu \mathrm{M}$ and NOC $10 \mu \mathrm{M}$ & 7 \\
48 & $7.08559516^{\mathrm{a}}$ & BNF $1 \mu \mathrm{M}$ and NOC $10 \mu \mathrm{M}$ & 7 \\
48 & $5.93579771^{\mathrm{a}}$ & BNF $1 \mu \mathrm{M}$ and NOC $10 \mu \mathrm{M}$ & 7 \\
72 & $0.66411867^{\mathrm{a}}$ & BNF $1 \mu \mathrm{M}$ and NOC $10 \mu \mathrm{M}$ & 7 \\
72 & $0.46601484^{\mathrm{a}}$ & BNF $1 \mu \mathrm{M}$ and NOC $10 \mu \mathrm{M}$ & 7 \\
72 & $1.65068662^{\mathrm{a}}$ & BNF $1 \mu \mathrm{M}$ and NOC $10 \mu \mathrm{M}$ & 7 \\
72 & $3.36291459^{\mathrm{a}}$ & BNF $1 \mu \mathrm{M}$ and NOC $10 \mu \mathrm{M}$ & 7 \\
\hline
\end{tabular}

${ }^{a}$ Data not included in the fit and used for validation. 
Table S2. Raw data from a dose response experiment with $\beta$-naphthoflavone (BNF) alone on ethoxyresorufin-O-deethylase (EROD) activities at 24 hours. Dimethylsulphoxide (DMSO) was used as in vehicle-control. Six different doses of BNF was tested 0.1, 0.3, 0.9, 3, 8 and 25 $\mu \mathrm{M}$. The data are originally presented in a diagram as mean values \pm standard deviation in Michelová M. Master-thesis, 2012 (reference 2).

\begin{tabular}{|c|c|c|}
\hline $\begin{array}{l}\text { Time } \\
\text { (h) }\end{array}$ & $\begin{array}{l}\text { EROD activity } \\
\left(\mathrm{pmol} \cdot(\mathrm{min} \cdot \mathrm{mg} \text { protein })^{-1}\right)\end{array}$ & Treatment \\
\hline 24 & 0.046551324 & DMSO \\
\hline 24 & 0.060342951 & DMSO \\
\hline 24 & 0.039346828 & DMSO \\
\hline 24 & 0.084340084 & DMSO \\
\hline 24 & 0.670409585 & BNF $0.1 \mu \mathrm{M}$ \\
\hline 24 & 0.674327937 & BNF $0.1 \mu \mathrm{M}$ \\
\hline 24 & 0.684057217 & BNF $0.1 \mu \mathrm{M}$ \\
\hline 24 & 0.730882299 & BNF $0.1 \mu \mathrm{M}$ \\
\hline 24 & 2.155646156 & BNF $0.3 \mu \mathrm{M}$ \\
\hline 24 & 2.998096302 & BNF $0.3 \mu \mathrm{M}$ \\
\hline 24 & 6.882963038 & BNF $0.3 \mu \mathrm{M}$ \\
\hline 24 & 7.118508328 & BNF $0.3 \mu \mathrm{M}$ \\
\hline 24 & 9.826610315 & BNF $0.9 \mu \mathrm{M}$ \\
\hline 24 & 9.640790055 & BNF $0.9 \mu \mathrm{M}$ \\
\hline 24 & 13.20528880 & BNF $0.9 \mu \mathrm{M}$ \\
\hline 24 & 15.66574736 & BNF $0.9 \mu \mathrm{M}$ \\
\hline 24 & 10,47750098 & $\mathrm{BNF} 1 \mu \mathrm{M}$ \\
\hline 24 & 15,75083724 & BNF $1 \mu \mathrm{M}$ \\
\hline 24 & 11,37043434 & $\mathrm{BNF} 1 \mu \mathrm{M}$ \\
\hline 24 & 11,46827036 & BNF $1 \mu \mathrm{M}$ \\
\hline
\end{tabular}


BNF $3 \mu \mathrm{M}$

BNF $3 \mu \mathrm{M}$

BNF $3 \mu \mathrm{M}$

24

14.37496398

BNF $3 \mu \mathrm{M}$

24

8.667026192

BNF $8 \mu \mathrm{M}$

24

12.63653731

BNF $8 \mu \mathrm{M}$

24

8.916652597

BNF $8 \mu \mathrm{M}$

24

11.58314181

BNF $8 \mu \mathrm{M}$

24

4.356204610

BNF $25 \mu \mathrm{M}$

24

7.245004896

BNF $25 \mu \mathrm{M}$

24

3.180976352

BNF $25 \mu \mathrm{M}$

24

5.051250052 
Table S3. Raw data from dose response experiment with nocodazole (NOC) alone on ethoxyresorufin- $O$-deethylase (EROD) activities at 6, 12, 24, 48 and 72h. Dimethylsulphoxide (DMSO) was used as vehicle-control. Three different doses of NOC was tested, 1, 10 1nd 25 $\mu \mathrm{M}$. No significant induction of CYP1A activities could be measured in cells treated with 1,10 or $25 \mu \mathrm{M}$ NOC alone compared to that in vehicle-control treated (DMSO) cells and no comparison with fitted values were made for those treatments. The data are originally presented in a diagram as mean values \pm standard deviation in Michelová M. Master-thesis, 2012 (reference 2).

\begin{tabular}{lll}
\hline $\begin{array}{l}\text { Time } \\
(\mathrm{h})\end{array}$ & $\begin{array}{l}\text { EROD activity } \\
\left(\text { pmol } \cdot(\mathrm{min} \cdot \mathrm{mg} \text { protein })^{-1}\right)\end{array}$ & Treatment \\
\hline 6 & 0.11377082 & DMSO \\
6 & 0.25665065 & DMSO \\
6 & 0.16774007 & DMSO \\
6 & 0.01560841 & DMSO \\
12 & 0.11538702 & DMSO \\
12 & 0.1016562 & DMSO \\
12 & 0.2325335 & DMSO \\
12 & 0.09802283 & DMSO \\
24 & -0.0063485 & DMSO \\
24 & 0.05044173 & DMSO \\
24 & -0.0005397 & DMSO \\
24 & -0.0105922 & DMSO \\
48 & -0.0274927 & DMSO \\
48 & 0.03330583 & DMSO \\
48 & 0.05984807 & DMSO \\
48 & -0.0017851 & DMSO \\
72 & 0.0029007 & DMSO \\
72 & 0.06646949 & DMSO \\
72 & 0.0272952 & DMSO \\
72 & 0.0057347 &
\end{tabular}




\begin{tabular}{|c|c|c|}
\hline 6 & 0.02561141 & NOC $1 \mu \mathrm{M}$ \\
\hline 6 & 0.03276828 & NOC $1 \mu \mathrm{M}$ \\
\hline 6 & 0.07516854 & NOC $1 \mu \mathrm{M}$ \\
\hline 6 & 0.24248221 & NOC $1 \mu \mathrm{M}$ \\
\hline 12 & -0.0246521 & NOC $1 \mu \mathrm{M}$ \\
\hline 12 & -0.0079321 & NOC $1 \mu \mathrm{M}$ \\
\hline 12 & 0.18523452 & NOC $1 \mu \mathrm{M}$ \\
\hline 12 & 0.18955516 & NOC $1 \mu \mathrm{M}$ \\
\hline 24 & 0.00743391 & NOC $1 \mu \mathrm{M}$ \\
\hline 24 & -0.0256403 & NOC $1 \mu \mathrm{M}$ \\
\hline 24 & -0.0031455 & NOC $1 \mu \mathrm{M}$ \\
\hline 24 & 0.03193376 & NOC $1 \mu \mathrm{M}$ \\
\hline 48 & -0.0303212 & NOC $1 \mu \mathrm{M}$ \\
\hline 48 & 0.04061289 & NOC $1 \mu \mathrm{M}$ \\
\hline 48 & 0.07123973 & NOC $1 \mu \mathrm{M}$ \\
\hline 48 & 0.0127288 & NOC $1 \mu \mathrm{M}$ \\
\hline 72 & 0.0027803 & NOC $1 \mu \mathrm{M}$ \\
\hline 72 & -0.0623571 & NOC $1 \mu \mathrm{M}$ \\
\hline 72 & 0.0249061 & NOC $1 \mu \mathrm{M}$ \\
\hline 72 & 0.0216266 & NOC $1 \mu \mathrm{M}$ \\
\hline 6 & 0.0763394 & NOC $10 \mu \mathrm{M}$ \\
\hline 6 & 0.04654934 & NOC $10 \mu \mathrm{M}$ \\
\hline 6 & 0.26674799 & NOC $10 \mu \mathrm{M}$ \\
\hline 6 & 0.06454803 & NOC $10 \mu \mathrm{M}$ \\
\hline 12 & 0.07035758 & NOC $10 \mu \mathrm{M}$ \\
\hline 12 & 0.05555987 & NOC $10 \mu \mathrm{M}$ \\
\hline 12 & 0.17346336 & $\mathrm{NOC} 10 \mu \mathrm{M}$ \\
\hline 12 & 0.20638625 & NOC $10 \mu \mathrm{M}$ \\
\hline 24 & 0.0681884 & NOC $10 \mu \mathrm{M}$ \\
\hline 24 & -0.0734886 & NOC $10 \mu \mathrm{M}$ \\
\hline 24 & 0.14514875 & NOC $10 \mu \mathrm{M}$ \\
\hline 24 & 0.10303029 & $\mathrm{NOC} 10 \mu \mathrm{M}$ \\
\hline
\end{tabular}


NOC $10 \mu \mathrm{M}$

NOC $10 \mu \mathrm{M}$

NOC $10 \mu \mathrm{M}$

NOC $10 \mu \mathrm{M}$

NOC $10 \mu \mathrm{M}$

NOC $10 \mu \mathrm{M}$

72

0.0459174

NOC $10 \mu \mathrm{M}$

NOC $10 \mu \mathrm{M}$

$\begin{array}{lll}6 & 0.00804636 & \text { NOC } 25 \mu \mathrm{M} \\ 6 & -0.0439197 & \text { NOC } 25 \mu \mathrm{M} \\ 6 & 0.27521007 & \text { NOC } 25 \mu \mathrm{M} \\ 6 & 0.15776044 & \text { NOC } 25 \mu \mathrm{M} \\ 12 & -0.1723871 & \text { NOC } 25 \mu \mathrm{M} \\ 12 & 0.06644439 & \text { NOC } 25 \mu \mathrm{M} \\ 12 & 0.12006505 & \text { NOC } 25 \mu \mathrm{M} \\ 12 & 0.06413321 & \text { NOC } 25 \mu \mathrm{M} \\ 24 & -0.0644523 & \text { NOC } 25 \mu \mathrm{M} \\ 24 & 0.00814383 & \text { NOC } 25 \mu \mathrm{M} \\ 24 & 0.10558674 & \text { NOC } 25 \mu \mathrm{M} \\ 24 & 0.01805869 & \text { NOC } 25 \mu \mathrm{M} \\ 48 & 0.16261439 & \text { NOC } 25 \mu \mathrm{M} \\ 48 & 0.09551795 & \text { NOC } 25 \mu \mathrm{M} \\ 48 & 0.03861299 & \text { NOC } 25 \mu \mathrm{M} \\ 48 & 0.00371416 & \text { NOC } 25 \mu \mathrm{M} \\ 72 & 0.09099329 & \text { NOC } 25 \mu \mathrm{M} \\ 72 & 0.15649775 & \text { NOC } 25 \mu \mathrm{M} \\ 72 & 0.0309007 & \text { NOC } 25 \mu \mathrm{M} \\ 72 & 0.06562328 & \text { NOC } 25 \mu \mathrm{M}\end{array}$




\section{FIGURES:}

Figure S1. Local Sensitivity Analysis. Effects of the ten parameters on the EROD activity of free CYP1A enzymes $\left(E_{f}^{E R O D}\right)$ as the model output. The model is described by equations (4), (5), (9)-(13) and the parameters are listed in Table 1 . The diagram shows sensitivity of $E_{f}^{E R O D}$ to small perturbations in the nominal values of the ten parameters. It shows that $k_{D}$ has the largest negative average effects on the $E_{f}^{E R O D}$ values whereas $k_{O X}$ has the least positive average influence.

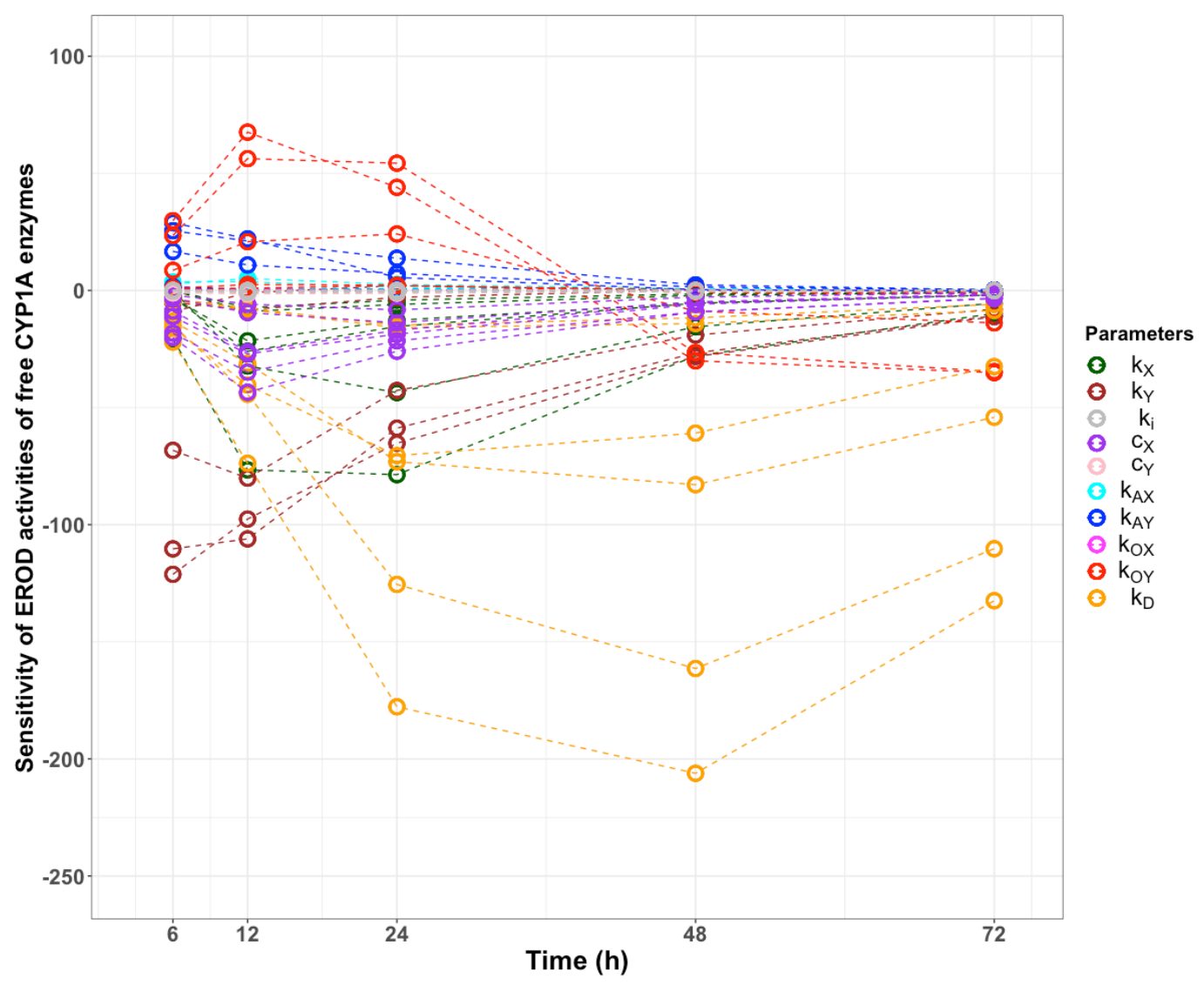


Figure S2. Collinearity index for all parameter combinations. The dashed red line denotes the threshold value of 20, for the collinearity index. All sets inholding different parameter combinations in the model have a collinearity index below 20 .

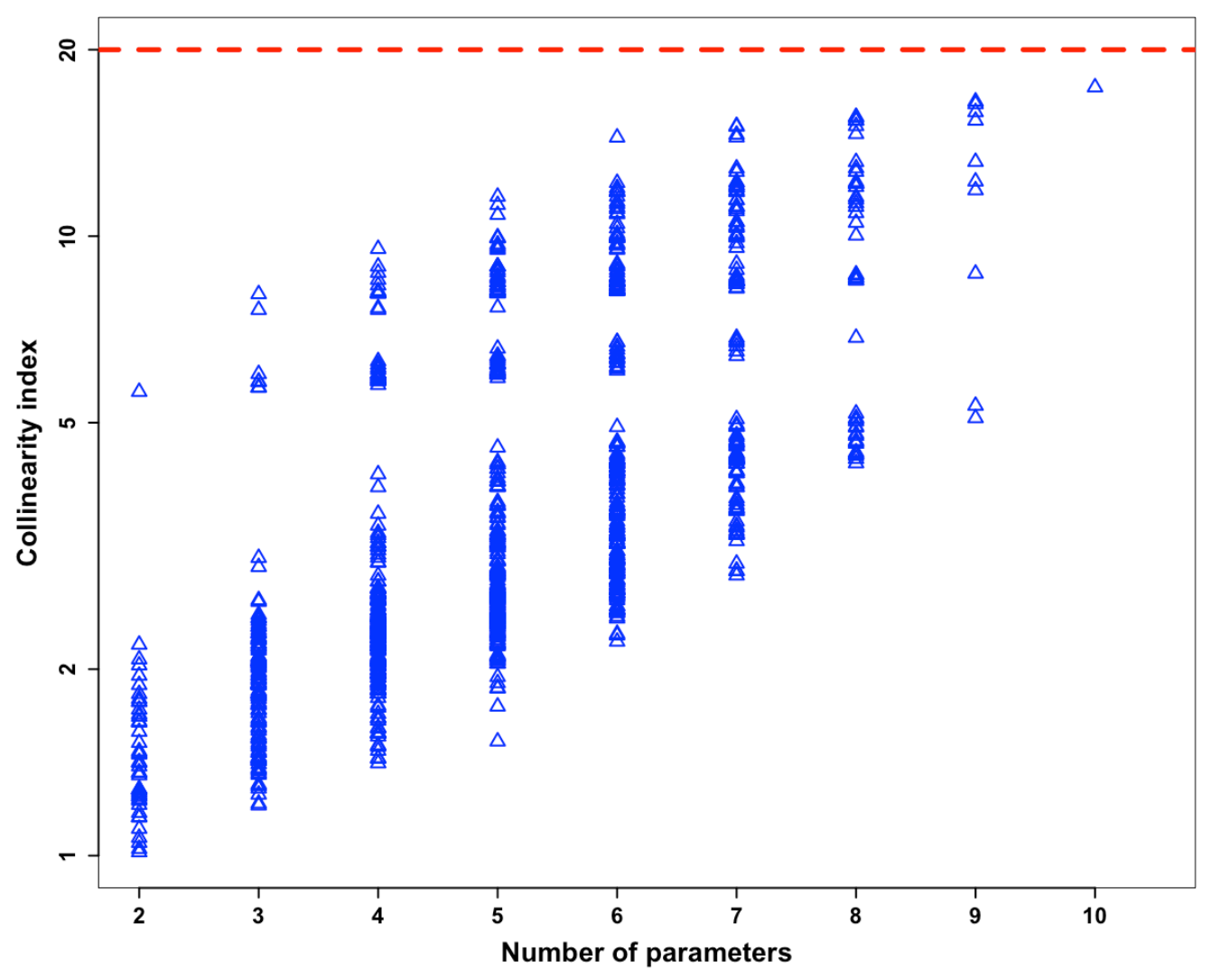




\section{REFERENCES}

(1) Gräns, J.; Johansson, J.; Michelová. M.; Wassmur, B.; Norström, E.; Wallin, M.;

Celander, M.C. Mixture effects between different azoles and $\beta$-naphthoflavone on the CYP1A biomarker in a fish cell line. Aquat. Toxicol. 2015. 164, 43-51.

(2) Michelová, M. Cocktail effects of $\beta$-naphthoflavone and nocodazole in the Poeciliopsis lucida cell line. 2012. Master-thesis. University of Gothenburg. 